\title{
Enhancement of local electric field in core-shell orientation of ellipsoidal metal/dielectric nanoparticles $\|^{*}$
}

\author{
A.A. Ismai 1 , A.V. Gholap ${ }^{1}$, Y.A. Abbo ${ }^{2}$ \\ 1 Department of Physics, Addis Ababa University, P O Box 1176, Addis Ababa, Ethiopia \\ 2 Department of Physics, Wollega University, P O Box 395, Nekemt, Ethiopia
}

Received October 20, 2016, in final form January 10, 2017

\begin{abstract}
In this paper it is shown that the enhancement factor of the local electric field in metal covered ellipsoidal nanoparticles embedded in a dielectric host matrix has two maxima at two different frequencies. The second maximum for the metal covered inclusions with large dielectric core (small metal fraction $p$ ) is comparatively large. This maximum strongly depends on the depolarization factor of the core $L_{z}^{(1)}$, keeping that of the shell $L_{z}^{(2)}$ constant and is less than $L_{z}^{(1)}$. If the frequency of the external radiation approaches the frequency of surface plasmons of a metal, the local field in the particle considerably increases. The importance of maximum value of enhancement factor $|A|^{2}$ of the ellipsoidal inclusion is emphasized in the case where the dielectric core exceeds metal fraction of the inclusion. The results of numerical computations for typical small silver particles are presented graphically.
\end{abstract}

Key words: enhancement factor, ellipsoidal nanoinclusion, depolarization factor, local field, resonant frequency

PACS: $42.65 . P c, 42.79 . T a, 78.67 . S c$, 78.67.-n

\section{Introduction}

The enhancement of the local electric field of the incident electromagnetic radiation in the composites of metal covered nanoparticles with dielectric core is of great importance due to different possible applications such as surface enhanced Raman spectroscopy [1], metal enhanced florescence [2], quantum electrodynamics [3, 4], nonlinear optical effect [5], quantum optomechanics [6], optical sensors [7] and nano-optical tweezers [8]. It is known that the local electric field in the inclusions can be considerably enhanced if a frequency of the incident radiation is close to the surface plasmon frequency [9]. This problem was studied in connection with the optically induced bistability [10, 11] and it is accepted that such an enhancement takes place only on one resonant frequency. It is clear that the nonlinear part of the dielectric function (DF) is important only if the electric fields are comparable with the inner atomic fields. At present, such fields may be achieved by laser radiation. Another interesting property of a pure metal and metal-covered dielectric small particles is an abnormal enhancement of the local field, when the frequency of the incident electromagnetic wave approaches the surface plasmon frequency of the metal [12]. The fact that the surface plasmon (SP) strongly depends on size, shape, distribution of metal nanoparticles as well as on the surrounding dielectric matrix offers an opportunity for manufacturing new promising nonlinear materials, nanodevices and optical elements. The ellipsoidal shape does represent the most general geometry suitable for many practical applications: in particular, it allows us to analyze two important limiting cases, namely the spherical and the cylindrical ones. The existence of a two-peak

\footnotetext{
*E-mail: abdulhayi.abdella@aau.edu.et
} 
value structure of the frequency dependence of the enhancement factor was first presented by Sisay and Mal'nev [13] for a composite with metal coated spherical nanoinclusions. Under this context, the present investigation provides a very general conceptual framework, including those specific cases previously investigated. A detailed theoretical and numerical analysis of the local field enhancement in small metal covered ellipsoidal inclusions in the electrostatic approximation is the aim of this study. In section 2 and 3, we analyze the distribution of electric potential in a coated ellipsoidal metal nanoparticle when the incident electric field is parallel to one of the ellipsoid axes ( $z$-axis), and the enhancement factor of local field inside a metal covered ellipsoidal dielectric core embedded into a dielectric matrix, respectively. Lastly, in section 4 , the results of numerical calculations are illustrated graphically. In the conclusion, we summarize the main results of the paper.

\section{Electric potentials distribution in a coated ellipsoidal metal nanopar- ticle}

The most general smooth particle (the one without edges or corners) of regular shape of an ellipsoidal coordinates [14], with semiaxes $a>b>c$ [figure 1] (a)], can be obtained by considering the surface which is specified by

$$
\frac{x^{2}}{a^{2}}+\frac{y^{2}}{b^{2}}+\frac{z^{2}}{c^{2}}=1
$$

without loss of generality, considering a family of curves defined by

$$
f(q) \equiv \frac{x^{2}}{a^{2}+q}+\frac{y^{2}}{b^{2}+q}+\frac{z^{2}}{c^{2}+q}-1=0
$$

for $q>-c^{2}, f(q)=0$ defines an ellipsoid.

Consider a confocal core-shell ellipsoid shown in figure 1(b), which can represent a wide range of shapes from disks to rods. The principal semiaxes are $a_{1}, b_{1}$, and $c_{1}$ for the core surface and $a_{2}, b_{2}$, and $c_{2}$ for the outer shell surface. Any confocal ellipsoidal surface can be expressed by

$$
\frac{x^{2}}{a_{1}^{2}+q}+\frac{y^{2}}{b_{1}^{2}+q}+\frac{z^{2}}{c_{1}^{2}+q}=1, \quad\left(a_{1}>b_{1}>c_{1}\right) .
$$

This equation, a cubic in $q$, has three real roots $\xi, \eta$, and $\zeta$ that define the ellipsoidal coordinates. The coordinate $\xi$ is normal to the surface. The variables $\eta$ and $\zeta$ are the parameters of confocal hyperboloids

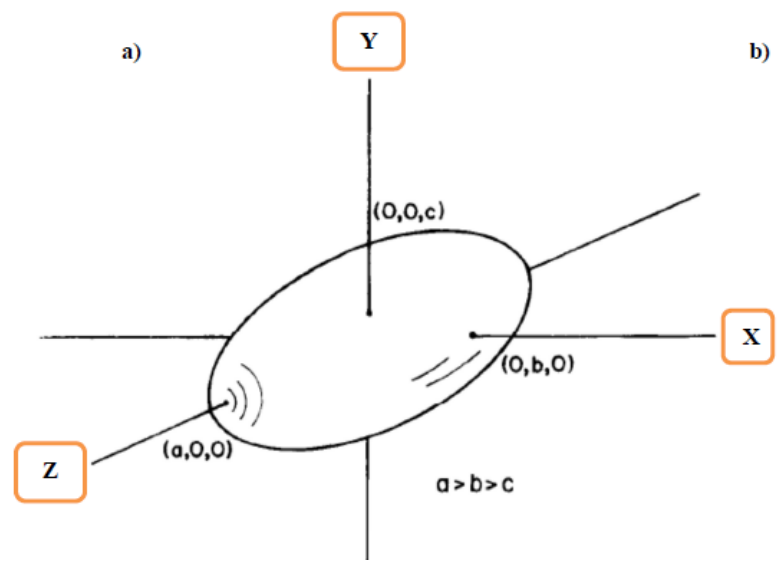

b)

Figure 1. (Color online) a) Ellipsoidal particle. b) Metal coated ellipsoidal particle with internal dielectric core (c), external shell (s) embedded into a given matrix (m). 
and as such serve to measure the position on any ellipsoid $\xi=$ constant. In other words, each ellipsoidal surface is defined by a constant $\xi$. Therefore, $\xi=0$ is the equation of the surface of inner ellipsoid and $\xi=t$ is that of the surface of the outer ellipsoid, where $a_{1}^{2}+t=a_{2}^{2}, b_{1}^{2}+t=b_{2}^{2}, c_{1}^{2}+t=c_{2}^{2}$. For a given $(x, y, z)$, if we assume $x>0, y>0, z>0$, there is a one to one correspondence between $(x, y, z)$ and the three largest roots $(\xi, \eta, \zeta)$. This implies that the transformation to rectangular coordinates is obtained by solving equation (2.2) simultaneously for $x, y, z$, which shows that an expression for the $z$ will be given as:

$$
z=\left[\frac{\left(c_{1}^{2}+\xi\right)\left(c_{1}^{2}+\eta\right)\left(c_{1}^{2}+\zeta\right)}{\left(a_{1}^{2}-c_{1}^{2}\right)\left(b_{1}^{2}-c_{1}^{2}\right)}\right]^{1 / 2} .
$$

We assume that the uniform electrostatic field $\mathbf{E}_{0}$ is directed along the $z$-axis. Then, the external field potential can be written in the form

$$
\Phi_{0}=-E_{0} z=-E_{0}\left[\frac{\left(c_{1}^{2}+\xi\right)\left(c_{1}^{2}+\eta\right)\left(c_{1}^{2}+\zeta\right)}{\left(a_{1}^{2}-c_{1}^{2}\right)\left(b_{1}^{2}-c_{1}^{2}\right)}\right]^{1 / 2}=-E_{0} F_{1}(\xi) G(\eta, \zeta),
$$

where we substitute $F_{1}(\xi)=\left(c_{1}^{2}+\xi\right)^{1 / 2}$ and $G(\eta, \zeta)=\left\{\left(c_{1}^{2}+\eta\right)\left(c_{1}^{2}+\zeta\right) /\left[\left(a_{1}^{2}-c_{1}^{2}\right)\left(b_{1}^{2}-c_{1}^{2}\right)\right]\right\}^{1 / 2}$ from equation (2.3).

Let us assume an electrostatic approximation in which the wavelength of the incident electromagnetic wave is much greater than the typical size of the inclusion. The distribution of electric potentials between the interfaces of an ellipsoidal metal coated nanoparticle can be expressed as: (i) $\Phi_{\mathrm{c}}$ in the dielectric core, (ii) $\Phi_{\mathrm{s}}$ in the metal cover shell and (iii) $\Phi_{\mathrm{m}}$ in the embedded dielectric matrix. Under the action of a constant external electric field $\mathbf{E}_{0}$, they can be described by the following expressions [15]

$$
\begin{aligned}
& \Phi_{\mathrm{c}}=K_{1} F_{1}(\xi) G(\eta, \zeta), \quad-c_{1}^{2}<\xi<0, \\
& \Phi_{\mathrm{s}}=\left[K_{2} F_{1}(\xi)+K_{3} F_{2}(\xi)\right] G(\eta, \zeta), \quad 0 \leqslant \xi<t, \\
& \Phi_{\mathrm{m}}=\Phi_{0}+\Phi_{\mathrm{p}}, \quad t \leqslant \xi<\infty,
\end{aligned}
$$

which are the solutions of the Laplace's equation in ellipsoidal coordinates stated as:

$$
\begin{aligned}
\nabla^{2} \Phi_{i}= & (\eta-\zeta) f(\xi) \frac{\partial}{\partial \xi}\left[f(\xi) \frac{\partial \Phi_{i}}{\partial \xi}\right]+(\zeta-\xi) f(\eta) \frac{\partial}{\partial \eta}\left[f(\eta) \frac{\partial \Phi_{i}}{\partial \eta}\right] \\
& +(\xi-\eta) f(\zeta) \frac{\partial}{\partial \zeta}\left[f(\zeta) \frac{\partial \Phi_{i}}{\partial \zeta}\right]=0,
\end{aligned}
$$

where $f(q)=\left[\left(a^{2}+q\right)\left(b^{2}+q\right)\left(c^{2}+q\right)\right]^{1 / 2}$, here $q$ stands for $\xi, \eta, \zeta$ in the function of $f(\xi), f(\eta), f(\zeta)$ of the ellipsoidal coordinates in equation 2.6 above. The subscript " $i$ " indicates the interface potentials in the dielectric core $i=\mathrm{c}$, metal shell $i=\mathrm{s}$, and in the host matrix $i=\mathrm{m}$, respectively. The potential $\Phi_{\mathrm{m}}$ in the surrounding medium of equation 2.5 is the sum of $\Phi_{0}$ and the perturbing potential $\Phi_{\mathrm{p}}$ of the particle which is given by

$$
\Phi_{\mathrm{p}}=K_{4} F_{2}(\xi) G(\eta, \zeta),
$$

where $F_{2}(\xi)=F_{1}(\xi) \int_{\xi}^{\infty} \mathrm{d} q /\left(c_{1}^{2}+q\right) f_{1}(q)$, with the property $\lim _{\xi \rightarrow \infty} F_{2}(\xi)=0 ; f_{1}(q)=\left[\left(a_{1}^{2}+q\right)\left(b_{1}^{2}+\right.\right.$ $\left.q)\left(c_{1}^{2}+q\right)\right]^{1 / 2}$, and $K_{1}, K_{2}, K_{3}, K_{4}$ are unknown constants, to be determined by the boundary conditions specified below. Therefore, the potential $\Phi_{\mathrm{m}}$, in the surrounding medium can be expressed by putting equations 2.4 and 2.7 in 2.5) as:

$$
\Phi_{\mathrm{m}}=\left[-E_{0} F_{1}(\xi)+K_{4} F_{2}(\xi)\right] G(\eta, \zeta), \quad t \leqslant \xi<\infty .
$$

The boundary conditions for the potentials can be found from the continuity conditions of the potentials themselves:

$$
\begin{aligned}
& \Phi_{\mathrm{c}}=\Phi_{\mathrm{s}} \quad \text { at } \quad \xi=0, \\
& \Phi_{\mathrm{s}}=\Phi_{\mathrm{m}} \quad \text { at } \quad \xi=t,
\end{aligned}
$$


and the normal components of the electric displacement vector:

$$
\begin{aligned}
& \varepsilon_{1} \frac{\partial \Phi_{\mathrm{c}}}{\partial \xi}=\varepsilon_{2} \frac{\partial \Phi_{\mathrm{s}}}{\partial \xi} \quad \text { at } \quad \xi=0, \\
& \varepsilon_{2} \frac{\partial \Phi_{\mathrm{s}}}{\partial \xi}=\varepsilon_{\mathrm{m}} \frac{\partial \Phi_{\mathrm{m}}}{\partial \xi} \quad \text { at } \quad \xi=t .
\end{aligned}
$$

The unknown coefficients $K_{1}, K_{2}, K_{3}, K_{4}$ can be determined by substituting expressions of equation 2.5) in the system of equations (2.8) and (2.9) at the boundaries of dielectric core-metal and metal-host matrix interfaces, and solving simultaneously, we obtain a system of linear algebraic equations as listed below:

$$
\begin{aligned}
& K_{1}=-\frac{\varepsilon_{\mathrm{m}} \varepsilon_{2}}{Q} E_{0}, \\
& K_{2}=-\frac{\varepsilon_{\mathrm{m}}\left[\left(\varepsilon_{1}-\varepsilon_{2}\right) L_{z}^{(1)}+\varepsilon_{2}\right]}{Q} E_{0}, \\
& K_{3}=\frac{a_{1} b_{1} c_{1} \varepsilon_{\mathrm{m}}\left(\varepsilon_{1}-\varepsilon_{2}\right)}{2 Q} E_{0}, \\
& K_{4}=-\frac{a_{2} b_{2} c_{2}}{2 Q}\left\{f\left(\varepsilon_{1}-\varepsilon_{2}\right)\left[L_{z}^{(2)}\left(\varepsilon_{2}-\varepsilon_{\mathrm{m}}\right)-\varepsilon_{2}\right]-\left(\varepsilon_{2}-\varepsilon_{\mathrm{m}}\right)\left[L_{z}^{(1)}\left(\varepsilon_{1}-\varepsilon_{2}\right)+\varepsilon_{2}\right]\right\} E_{0},
\end{aligned}
$$

where $Q=p \Delta$, and

$$
\Delta=\varepsilon_{2}^{2}\left[1+\frac{L_{z}^{(2)}-L_{z}^{(1)}}{p}\right]-q \varepsilon_{2}+\varepsilon_{1} \varepsilon_{\mathrm{m}} .
$$

Here, $q=\varepsilon_{1}\left[1-L_{z}^{(1)} / p\right]+\varepsilon_{\mathrm{m}}\left\{\left[L_{z}^{(2)}-1\right] / p+1\right\}$, and $p=f L_{z}^{(2)}\left[L_{z}^{(2)}-1\right]-L_{z}^{(1)}\left[L_{z}^{(2)}-1\right]$ is a metal fraction in the inclusion which is expressed by the volume fraction $f=a_{1} b_{1} c_{1} /\left(a_{2} b_{2} c_{2}\right)$ of the core into the whole inclusion, that is, the fraction of the total particle of the volume occupied by the inner ellipsoid. The variables $L_{z}^{(1)}$ and $L_{z}^{(2)}$ are the geometrical factors for the inner and outer confocal ellipsoids, and, $\varepsilon_{1}$, $\varepsilon_{2}$, and $\varepsilon_{\mathrm{m}}$ are the dielectric functions (DFs) of the core, metal shell, and the host matrix (the surrounding medium), respectively. Since we assume that a uniform, parallel electric field $\mathbf{E}_{0}$ is directed along the $z$-axis, and is thus along the major semi-axis of the ellipsoid $(a)$, the local field $E_{\text {loc }}$ in the dielectric core of the inclusion can be obtained with the help of the relation, $E_{\mathrm{loc}}=-\nabla \Phi$. The corresponding local fields in each portion between the interfaces are; $E_{\text {loc }}^{\mathrm{c}}$ of the core, $E_{\mathrm{loc}}^{\mathrm{s}}$ of the shell and $E_{\mathrm{loc}}^{\mathrm{m}}$ of the surrounding medium of metal coated inclusion. They can be presented with the relation

$$
\begin{aligned}
& E_{\mathrm{loc}}^{\mathrm{c}}=-\nabla \Phi_{\mathrm{c}}=A E_{0}, \\
& E_{\mathrm{loc}}^{\mathrm{s}}=-\nabla \Phi_{\mathrm{s}}=B E_{0}+C E_{0}, \\
& E_{\mathrm{loc}}^{\mathrm{m}}=-\nabla \Phi_{\mathrm{m}}=E_{0}+D E_{0},
\end{aligned}
$$

the factors that relate the local fields with the external incident electric field between the interfaces are given as below:

$$
\begin{aligned}
& A=\frac{\varepsilon_{2} \varepsilon_{\mathrm{m}}}{Q}, \\
& B=\frac{\varepsilon_{\mathrm{m}}\left[\left(\varepsilon_{1}-\varepsilon_{2}\right) L_{z}^{(1)}+\varepsilon_{2}\right]}{Q}, \\
& C=-\frac{a_{1} b_{1} c_{1} \varepsilon_{\mathrm{m}}\left(\varepsilon_{1}-\varepsilon_{2}\right)}{2 Q} \int_{\xi}^{\infty} \frac{\mathrm{d} q}{\left(c_{1}^{2}+q\right) f_{1}(q)}, \\
& D=\frac{a_{2} b_{2} c_{2}}{2 Q}\left[\left(\varepsilon_{2}-\varepsilon_{\mathrm{m}}\right)\left\{\varepsilon_{2}+\left(\varepsilon_{1}-\varepsilon_{2}\right)\left[L_{z}^{(1)}-f L_{z}^{(2)}\right]\right\}+f \varepsilon_{2}\left(\varepsilon_{1}-\varepsilon_{2}\right)\right] \int_{\xi}^{\infty} \frac{\mathrm{d} q}{\left(c_{1}^{2}+q\right) f_{1}(q)} .
\end{aligned}
$$


It is important to remark that the external field is completely controlled by the coefficient $K_{4}$ (or/and $D$ ). At sufficiently large distances from the particle, the perturbing potential in equation (2.7) is negligible i.e., when $\xi \gg a_{2}^{2}$, therefore, we require that $\lim _{\xi \rightarrow \infty} \Phi_{\mathrm{p}}=0$. We note that at distances $r$ from the origin which are much greater than the largest semi-axis of the shell $a_{2}$ to any point on the ellipsoid $\xi$, then $x^{2}+y^{2}+z^{2}=\xi \simeq r^{2}$; the integral in equation (2.7), that can enter the constant $D$ of expression in equation (2.21) is approximately:

$$
\int_{\xi}^{\infty} \frac{\mathrm{d} \xi}{\left(c_{1}^{2}+\xi\right) f_{1}(\xi)} \simeq \int_{\xi}^{\infty} \frac{\mathrm{d} \xi}{\xi^{5 / 2}}=\frac{2}{3} \xi^{-3 / 2}, \quad\left(\xi \simeq r^{2} \gg a_{2}^{2}\right),
$$

and, therefore, the potential $\Phi_{\mathrm{p}}$ is given

$$
\Phi_{\mathrm{p}} \sim \frac{E_{0} \cos \theta}{r^{2}} \frac{a_{2} b_{2} c_{2}}{3 Q}\left[\left(\varepsilon_{2}-\varepsilon_{\mathrm{m}}\right)\left\{\varepsilon_{2}+\left(\varepsilon_{1}-\varepsilon_{2}\right)\left[L_{z}^{(1)}-f L_{z}^{(2)}\right]\right\}+f\left(\varepsilon_{1}-\varepsilon_{2}\right) \varepsilon_{2}\right], \quad\left(r \gg a_{2}\right),
$$

since the potential of ideal dipole is given by $\Phi=P \cos \theta /\left(4 \pi \varepsilon_{\mathrm{m}} r^{2}\right)$, we can recognize the equation 2.23) as the potential of a dipole with the moment

$$
P=4 \pi \varepsilon_{\mathrm{m}} \frac{a_{2} b_{2} c_{2}}{3 Q}\left[\left(\varepsilon_{2}-\varepsilon_{\mathrm{m}}\right)\left\{\varepsilon_{2}+\left(\varepsilon_{1}-\varepsilon_{2}\right)\left[L_{z}^{(1)}-f L_{z}^{(2)}\right]\right\}+f\left(\varepsilon_{1}-\varepsilon_{2}\right) \varepsilon_{2}\right]
$$

Therefore, this yields the polarizability

$$
\alpha_{z}=\frac{v\left[\left(\varepsilon_{2}-\varepsilon_{\mathrm{m}}\right)\left\{\varepsilon_{2}+\left(\varepsilon_{1}-\varepsilon_{2}\right)\left[L_{z}^{(1)}-f L_{z}^{(2)}\right]\right\}+f\left(\varepsilon_{1}-\varepsilon_{2}\right) \varepsilon_{2}\right]}{Q},
$$

where $v=4 \pi a_{2} b_{2} c_{2} / 3$ is the volume of the particle, and $f=a_{1} b_{1} c_{1} /\left(a_{2} b_{2} c_{2}\right)$ is the volume fraction. Here, it may be mentioned that, letting $q=\xi+t$ to solve the integral that will be substituted by the geometrical factors of the depolarization which is given by

$$
L_{z}^{k}=\frac{a_{k} b_{k} c_{k}}{2} \int_{0}^{\infty} \frac{\mathrm{d} q}{\left(c_{k}^{2}+q\right) f_{k}(q)}, \quad(k=1,2),
$$

the expression in equation (2.25) is equivalent to equation (2.21). Therefore, equation 2.21 coincides with the corresponding result shown in [15] for the polarizability of a coated ellipsoid. The coefficients $A, B, C, D$ are consistent with the coated sphere that can be verified by putting $L_{z}^{(1)}=L_{z}^{(2)}=1 / 3$, $Q=2 p \Delta / 9$, as shown by Sisay and Mal'nev [13].

\section{Resonant frequencies and enhancement factor of local field in metal covered ellipsoidal inclusion}

Among equations $2.18-(2.21$, we need only the coefficients $A$ and $D$ that enter the potential of the local field in the inclusion "core" and the induced dipole moment of the inclusion. Let us consider that the dielectric function of metal $\varepsilon_{2}$ is chosen to be in Drude form [16],

$$
\varepsilon_{2}=\varepsilon_{\infty}-\frac{1}{z(z+\mathrm{i} \gamma)}
$$

Here, we introduced dimensionless frequencies $z=\frac{\omega}{\omega_{\mathrm{p}}}$, and $\gamma=\frac{v}{\omega_{\mathrm{p}}}\left(\omega\right.$ and $\omega_{\mathrm{p}}$ are the frequency of the incident radiation and the plasma frequency of the metal shell, respectively; $v$ is the electron collision frequency). $\varepsilon_{\infty}$ is a constant that can be a function of the frequency and depends on the type of a metal. The real $\varepsilon_{2}^{\prime}$ and imaginary $\varepsilon_{2}^{\prime \prime}$ parts of dielectric function are given by

$$
\varepsilon_{2}^{\prime}=\varepsilon_{\infty}^{\prime}-\frac{1}{z^{2}+\gamma^{2}} \quad \text { and } \quad \varepsilon_{2}^{\prime \prime}=\varepsilon_{\infty}^{\prime \prime}+\frac{\gamma}{z\left(z^{2}+\gamma^{2}\right)} .
$$


The dielectric function of the inclusion core $\varepsilon_{1}$, in general case, includes a non-linear part with respect to the local field.

$$
\varepsilon_{1}=\varepsilon_{10}+\chi|\mathbf{E}|^{2}
$$

where $\varepsilon_{10}$ is the linear part of DF, $\chi$ is the nonlinear Kerr coefficient, $|\mathbf{E}|$ is the local field in the core. For week incident fields $|\mathbf{E}| \ll \varepsilon_{10}$, the local field is presented as in equation $2.15 E_{\mathrm{loc}}^{\mathrm{c}}=A E_{0}$. We call $A$ the enhancement factor, which is in general a complex function. It would be convenient to deal with the real quantity $|A|^{2}$, which can be presented as follows

$$
|A|^{2}=\frac{\varepsilon_{\mathrm{m}}^{2}}{p^{2}} \frac{\varepsilon_{2}^{\prime 2}+\varepsilon_{2}^{\prime \prime 2}}{\left\{\left(\varepsilon_{2}^{\prime 2}-\varepsilon_{2}^{\prime \prime 2}\right)\left[1+\frac{L_{z}^{(2)}-L_{z}^{(1)}}{p}\right]-q \varepsilon_{2}^{\prime}+\varepsilon_{1} \varepsilon_{\mathrm{m}}\right\}^{2}+\varepsilon_{2}^{\prime \prime 2}\left\{2 \varepsilon_{2}^{\prime}\left[1+\frac{L_{z}^{(2)}-L_{z}^{(1)}}{p}\right]-q\right\}^{2}} .
$$

For the sake of simplicity, we ignore the imaginary parts of $\varepsilon_{1}$ and $\varepsilon_{\mathrm{m}}$.

For an analytic analysis, let us consider an ideal case when a decay of the plasma vibrations is extremely small $\gamma \ll 1$. In this case, the second term in the denominator of equation (3.4) is proportional to $\varepsilon_{2}^{\prime \prime 2} \sim \gamma^{2}$ which is very small. Therefore, maximum of the enhancement factor $|A|^{2}$ corresponds to zero of the first term in the denominator of 3.4. This condition gives the quadratic equation with respect to $\varepsilon_{2}^{\prime}$

$$
\varepsilon_{2}^{\prime 2}\left[1+\frac{L_{z}^{(2)}-L_{z}^{(1)}}{p}\right]-q \varepsilon_{2}^{\prime}+\varepsilon_{1} \varepsilon_{\mathrm{m}}=0 .
$$

It has two roots, and we obtain two different resonant frequencies $z_{\mathrm{r}}$. In the real inclusions, $\gamma$ is not extremely small but finite. The behavior of $|A|^{2}$ as a function $z$ in this case can be analyzed only numerically. The results of this study are presented in the following section.

\section{Numerical results and discussion}

We start our numerical calculations with the enhancement factor of a pure metal particle $\left|A_{\mathrm{m}}\right|^{2}$. The numerical values of the dielectric functions of the composite used in this section are taken from $\left[12,[13,16] .\left|A_{\mathrm{m}}\right|^{2}\right.$ can be obtained from equation $\sqrt{2.18}$ by setting $p=1$ and making substitution $\varepsilon_{1} \rightarrow \varepsilon_{2}$, and $L_{z}^{(1)}=L_{z}^{(2)}=L$ as well

$$
\left|A_{\mathrm{m}}\right|^{2}=\frac{\varepsilon_{\mathrm{m}}^{2}}{\left[L\left(\varepsilon_{2}^{\prime}-\varepsilon_{\mathrm{m}}\right)+\varepsilon_{\mathrm{m}}\right]^{2}+L^{2} \varepsilon_{2}^{\prime \prime 2}},
$$

where the subscript " $\mathrm{m}$ " indicates pure metal inclusion. In figure 2, we present this quantity versus $z$, and one can see that the enhancement factor $\left|A_{\mathrm{m}}\right|^{2}$ in the physically interesting range of parameters sharply depends on the frequency of an incident electromagnetic wave $\omega$ and weakly depends on the depolarization factor decreasing with $L$. It can easily be seen from equation 4.1 that $\left|A_{\mathrm{m}}\right|^{2}=1$ as $L \rightarrow 0$. The maximum value of $\left|A_{\mathrm{m}}\right|^{2}$ at the constant values used for numerical calculations of the particle and the host matrix [12] is around 250.

As it is shown in figure 3 , by changing $\varepsilon_{\mathrm{m}}$ and $L$, one can obtain even larger $\left|A_{\mathrm{m}}\right|^{2}$ which is around 500. This means that, at comparatively large applied fields $E_{0}$ in the vicinity of the corresponding plasma resonance, it is necessary to consider the nonlinear terms in the dielectric function of equation (3.3). Further, we will compare it with $|A|^{2}$ of metal covered inclusions with different dielectric cores. This quantity is calculated with the help of the enhancement factor of equation (3.4) with typical numerical values of the dielectric functions of a composite.

Figures 47 show the dependence of $|A|^{2}$ on the depolarization factor of the shell and the core at different metal fraction $(p)$, respectively. Keeping the minimum value of the depolarization factor of the core constant, it is observed that the enhancement factor $|A|^{2}$ weakly depends on the depolarization factor of the shell decreasing with $L_{z}^{(2)}$, and the second maximum will appear as shown in figure 4 . At 


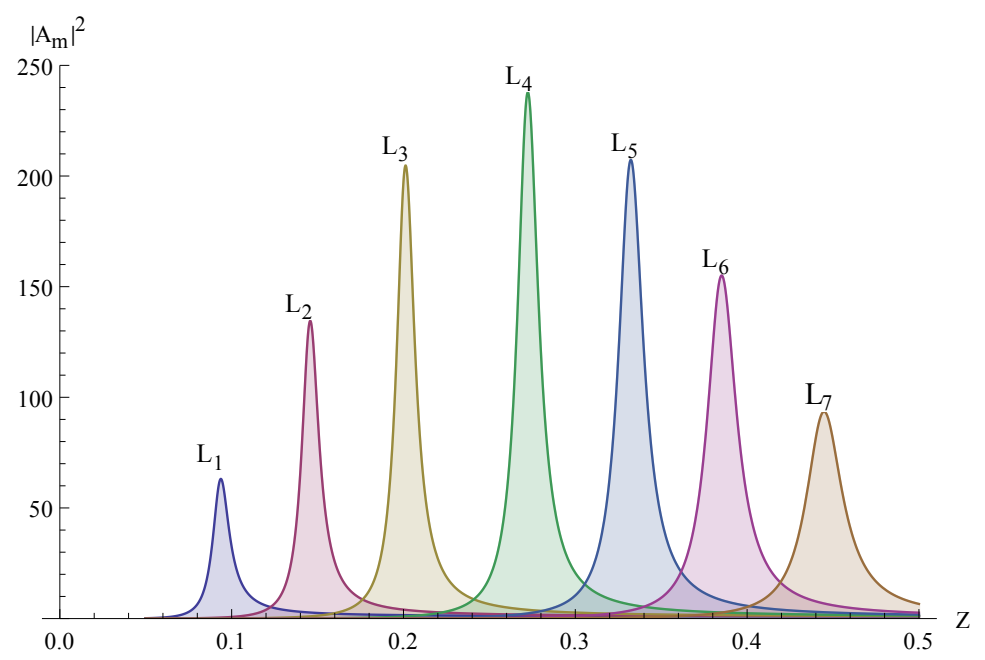

Figure 2. (Color online) The enhancement factor $\left|A_{\mathrm{m}}\right|^{2}$ for a small silver particle versus a function of the dimensionless frequency $z$ at different $L\left(L_{1}=0.02 ; L_{2}=0.05 ; L_{3}=0.1, L_{4}=0.2, L_{5}=0.33\right.$, $\left.L_{6}=0.5, L_{7}=0.8\right)$ with parameters of the particle $\varepsilon_{\mathrm{m}}=2.25, \varepsilon_{\infty}^{\prime}=4.5, \varepsilon_{\infty}^{\prime \prime}=0.16, \omega_{\mathrm{p}}=1.46 \cdot 10^{16}$, $v=1.68 \cdot 10^{14}, \gamma=1.15 \cdot 10^{-2}$.

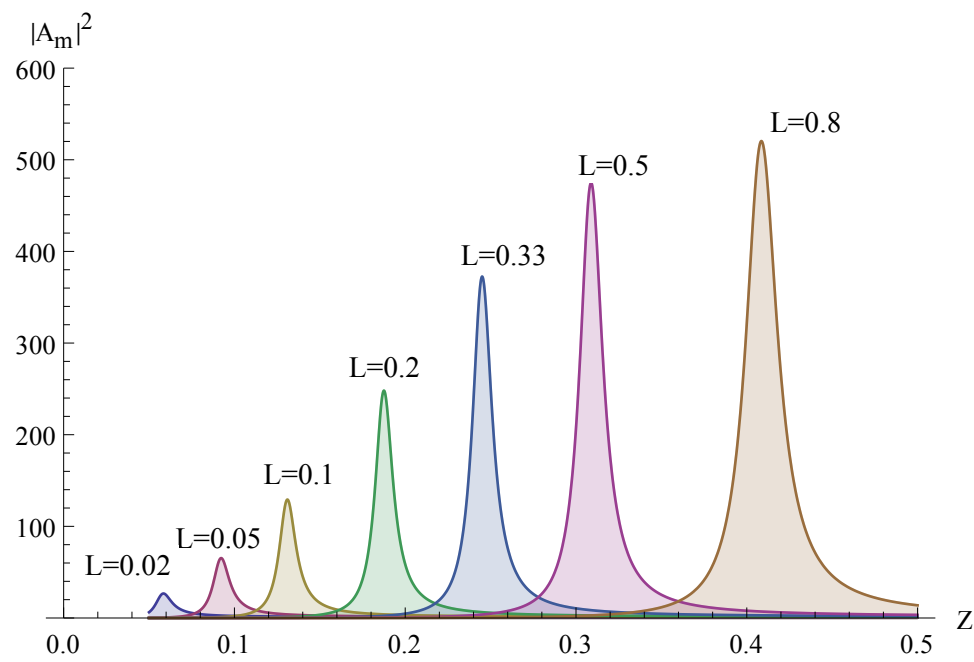

Figure 3. (Color online) The enhancement factor $\left|A_{\mathrm{m}}\right|^{2}$ for a small silver particle versus a function of the dimensionless frequency $z$ at different $L$ similar to figure 2 but with $\varepsilon_{\mathrm{m}}=6$.

this instant, the extent of $|A|^{2}$ value for the small core (large $p$ ) becomes comparable with the large core (small $p$ ) of the inclusion. When the maximum value of the core depolarization factor is kept at constant, as seen from figure 5 , the second maximum of the $|A|^{2}$ is dominant but weakly depends on depolarization factor of the shell, decreasing with $L_{z}^{(2)}$ which in turn leaves the appearance on the first maxima for the thin metal fraction of the inclusions.

Inspecting graphs in figure 6 , one can find that the second maximum of the enhancement factor $|A|^{2}$ strongly depends on $L_{z}^{(1)}$ suppressing the result of the first maximum that corresponds to a small core (large $p$ ) which leads to its disappearance at $L_{z}^{(1)}=0.8$ resulting in the $|A|^{2}$ value around 650 for a small metal fraction.

When $L_{z}^{(2)}=0.8$ is kept constant, the dependance of the second maximum on the depolarization factor of the core is not important, while the first maximum increases with $L_{z}^{(1)}$ as shown in figure 7. Here, it should be emphasized that, unlike spherical case [13], ellipsoidal inclusions having large dielectric core 

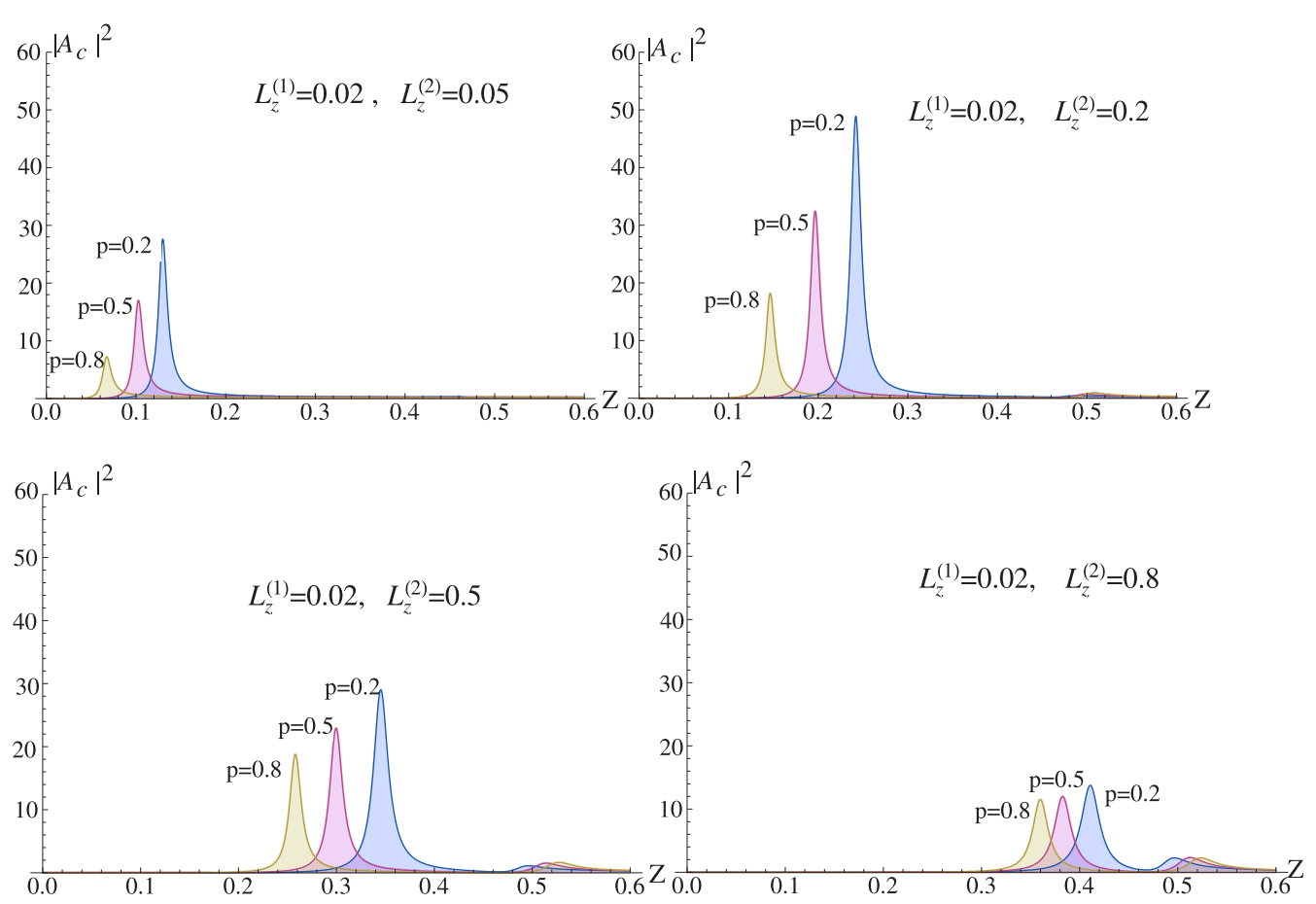

Figure 4. (Color online) The enhancement factor $\left|A_{\mathrm{c}}\right|^{2}$ for a silver ellipsoidal coated nanoparticle versus $z$ for $L_{z}^{(1)}$ (constant but minimum) $<L_{z}^{(2)}$ (variable), at $p=0.2,0.5,0.8$. Here and further we use the following parameters: $\varepsilon_{\infty}=4.5, \varepsilon_{1}=6, \varepsilon_{\mathrm{m}}=2.25$.
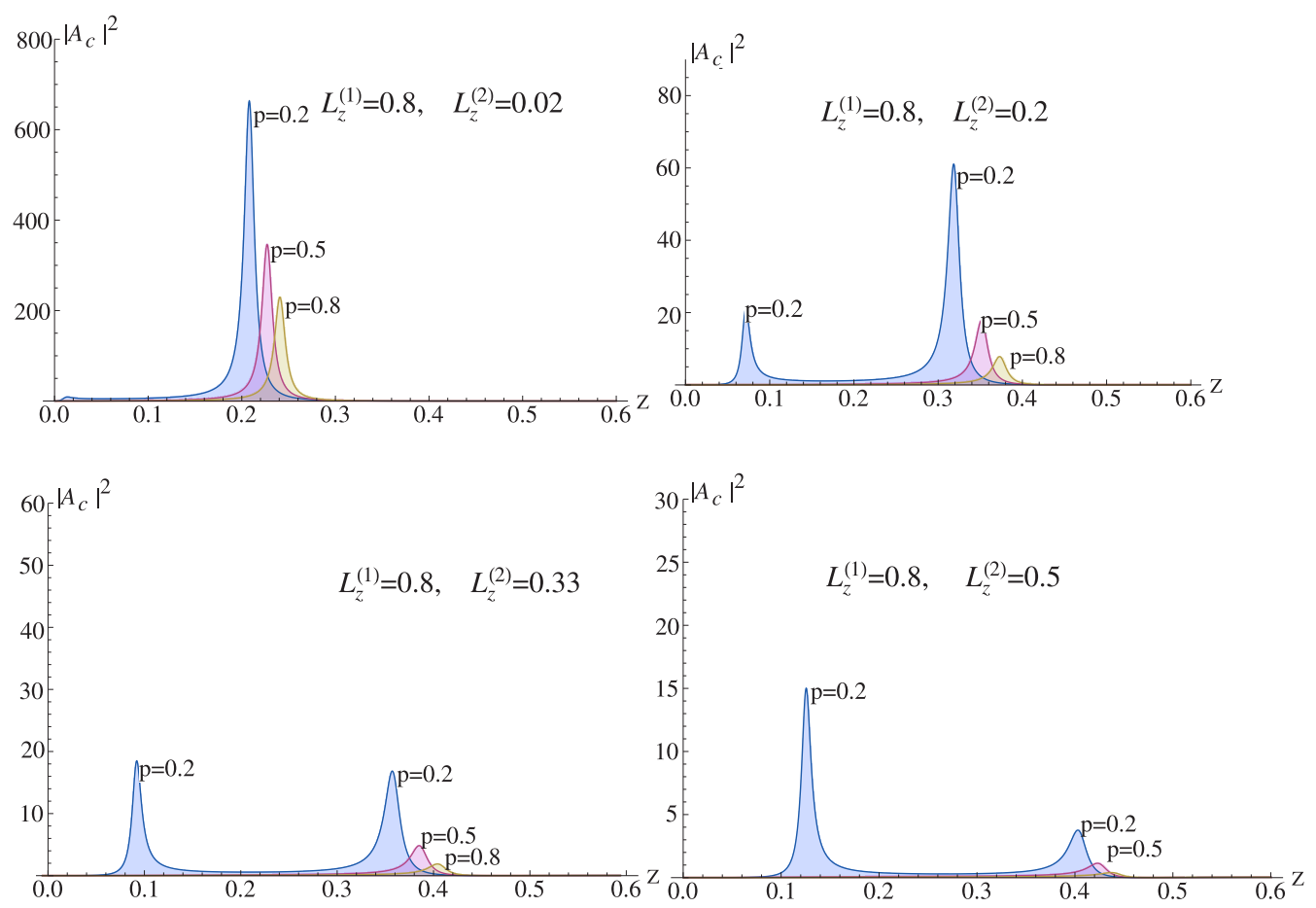

Figure 5. (Color online) The enhancement factor $\left|A_{\mathrm{c}}\right|^{2}$ for a silver ellipsoidal coated nanoparticle versus $z$ for $L_{z}^{(1)}$ (constant but maximum) $>L_{z}^{(2)}$ (variable), at $p=0.2,0.5,0.8$. 

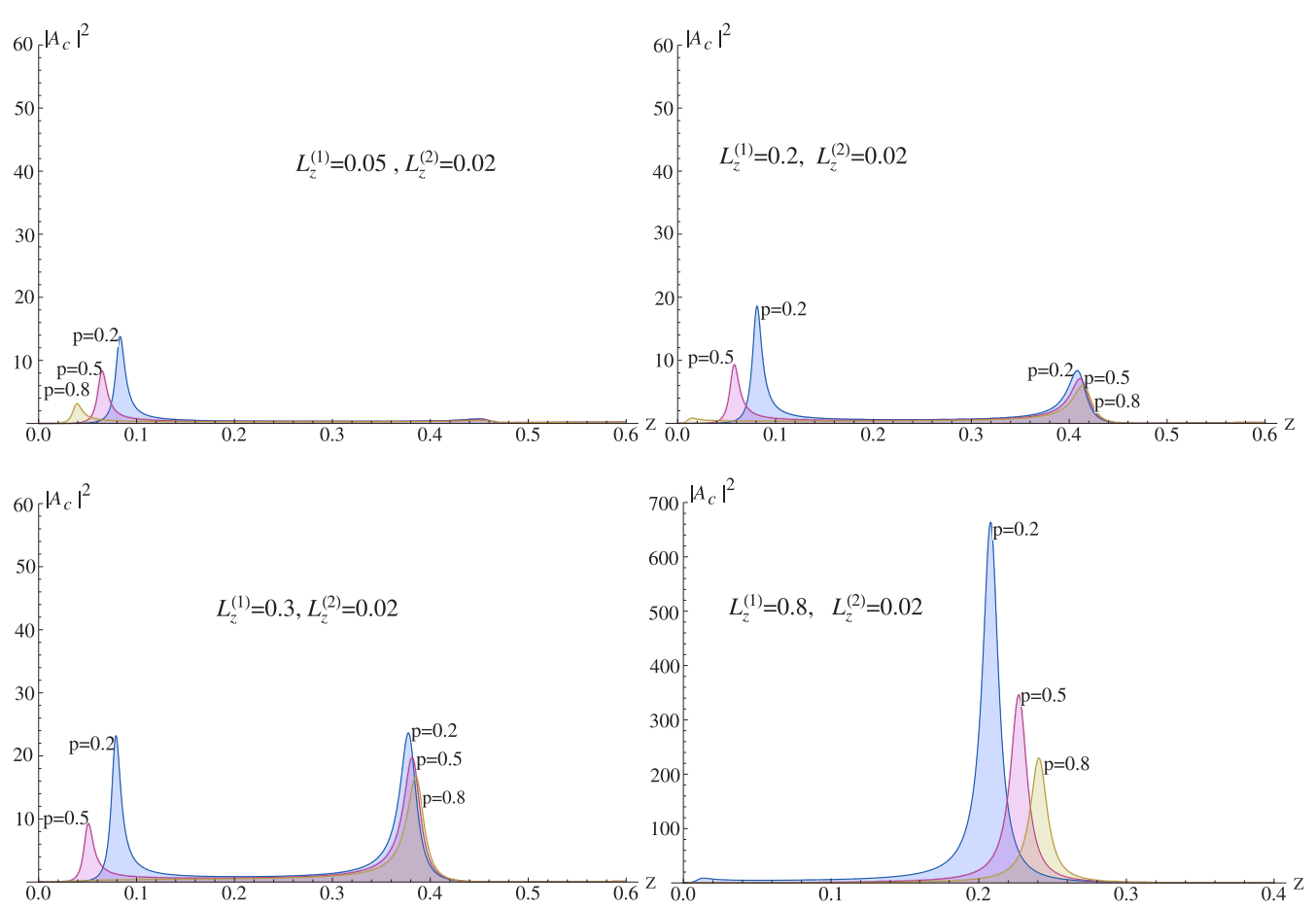

Figure 6. (Color online) The enhancement factor $\left|A_{\mathrm{c}}\right|^{2}$ for a silver ellipsoidal coated nanoparticle versus $z$ for $L_{z}^{(2)}$ (constant but minimum) $<L_{z}^{(1)}$ (variable).
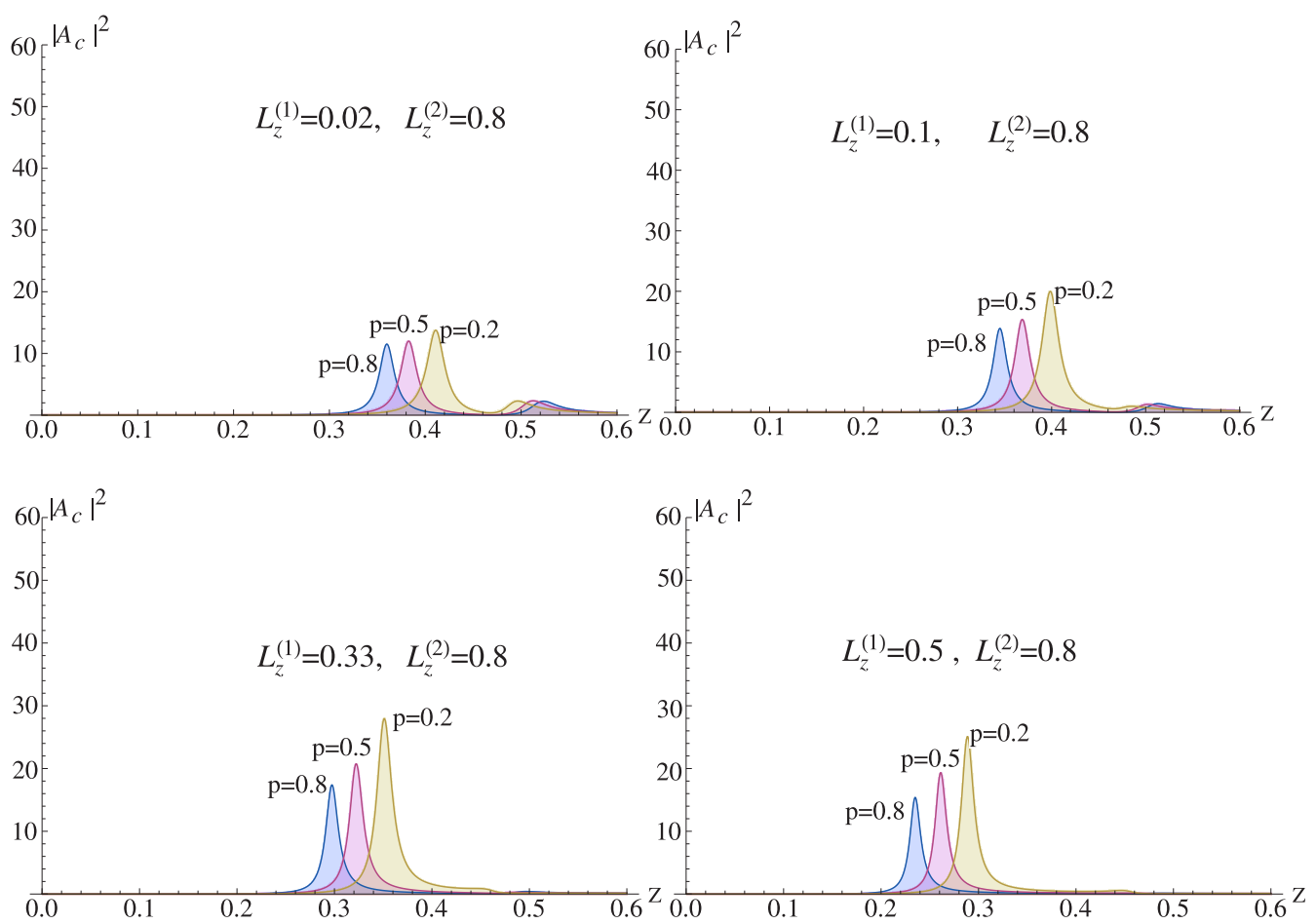

Figure 7. (Color online) The enhancement factor $\left|A_{\mathrm{c}}\right|^{2}$ for a silver ellipsoidal coated nanoparticle versus $z$ for $L_{z}^{(2)}$ (constant but maximum) $>L_{z}^{(1)}$ (variable), at $p=0.2,0.5,0.8$. 
that exceeds the fraction of metal ( $\operatorname{small} p$ ), the maximum value of $|A|^{2}$ is important depending on the suitable change of the depolarization factor of the shell and the core. This leads us to speak further about the composites of metal covered inclusions but not about the composites of dielectric inclusions having a metal core. Thus, we may presumably say that composites of metal covered inclusions behave in a manner different from the dielectric inclusions having a metal core.

\section{Conclusion}

In this paper, we have shown that depolarization factors $L_{z}^{(1)}$ of the core and $L_{z}^{(2)}$ of the shell are the only factor that determines the magnitude of the enhancement factor. The enhancement factor of the local field in a metal covered ellipsoidal inclusion with dielectric core in a linear host matrix has two maxima at two different frequencies that depends on the value of the core $L_{z}^{(1)}$ and the shell $L_{z}^{(2)}$ depolarization factor. It may be noted that in sphere the maxima are important in inclusions having large fraction of metal ( $p=0.9)$ that exceeds the fraction of the dielectric core, while in our case, the maxima are important when the metal fraction $(p=0.2)$ of ellipsoidal particle is very small.

\section{Acknowledgements}

This work is dedicated to the late Professor V.N. Mal'nev who departed suddenly on January 22, 2015. We greatly acknowledge his invaluable contributions right from problem setting to almost its conclusion. Let his soul rest in peace.

\section{References}

1. Prokes S.M., Glembocki O.J., Rendell R.W., Ancona M.G., Appl. Phys. Lett., 2007, 90, 093105, doi $10.1063 / 1.2709996$

2. Liu Y., Wang Sh., Park Y.-Sh., Yin X., Zhang X., Opt. Express, 2010, 18, 25029, doi 10.1364/OE.18.025029

3. Quan Q., Bulu I., Lončar M., Phys. Rev. A, 2009, 80, 011810, doi:10.1103/PhysRevA.80.011810

4. Russell K.J., Liu T.-L., Cui S., Hu E.L., Nat. Photonics, 2012, 6, 459-462, doi:10.1038/nphoton.2012.112

5. Spillane S., Kippenberg T., Vahala K., Nature, 2002, 415, 621-623, doi $10.1038 / 415621 \mathrm{a}$

6. Van Thourhout D., Roels J., Nat. Photonics, 2010, 4, 211-217, doi: $10.1038 /$ nphoton.2010.72

7. Anker J.N., Hall W.P., Lyandres O., Shah N.C., Zhao J., Van Duyne R.P., Nat. Mater., 2008, 7, 442-453, doi $10.1038 / \mathrm{nmat} 2162$

8. Juan M.L., Righini M., Quidant R., Nat. Photonics, 2011, 5, 349-356, doi 10.1038/nphoton.2011.56

9. Neeves A.E., Birnboim M.H., J. Opt. Soc. Am. B: Opt. Phys., 1989, 6, 787, doi:10.1364/JOSAB.6.000787

10. Kalyaniwalla N., Haus J.W., Inguva R., Birnboim M.H., Phys. Rev. A, 1990, 42, 5613, doi $10.1103 /$ PhysRevA.42.5613

11. Haraguchi M., Okamoto T., Inoue T., Nakagaki M., Koizumi H., Yamaguchi K., Lai C., Fukui M., Kamano M., Fujii M., IEEE J. Sel. Top. Quantum Electron., 2008, 14, No. 6, 1540, doi 10.1109/JSTQE.2008.917030

12. Buryi O.A., Grechko L.G., Mal'nev V.N., Shewamare S., Ukr. J. Phys., 2011, 56, 311.

13. Shewamare S., Mal'nev V.N., Physica B, 2012, 407, 4837-4842, doi 10.1016/j.physb.2012.08.007

14. Wikipedia, Ellipsoidal coordinates - Wikipedia, the free encyclopedia, 2016, [Online; accessed 10-Jan-2017], URL https://en.wikipedia.org/w/index.php?title=Ellipsoidal_coordinates\&oldid=722351999

15. Bohren C.F., Huffman D.R., Absorption and Scattering of Light by Small Particles, John Wiley, New York, 1983.

16. Abbo Y.A., Mal'nev V.N., Ismail A.A., Condens. Matter Phys., 2016, 19, No. 3, 33401, doi $10.5488 /$ CMP.19.33401 


\title{
Підсилення локального електричного поля в текстурі кор-оболонка еліпсоїдних наночастинок метал/діелектрик
}

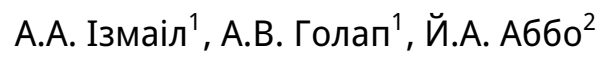 \\ 1 Фізичний факультет, університет Аддіс-Абеби, м. Аддіс-Абеба, Ефіопія \\ 2 Фізичний факультет, університет Уоллега, Р О Вох 395, Некемте, Ефіопія
}

В цій статті показано, що коефіцієнт підсилення локального електричного поля в еліпсоїдних частинках з металевим покриттям, вставлених в діелектричну матрицю, має два максимуми при різних частотах. Другий максимум для включень 3 металічним покриттям з великим діелектричним кором (мала фракція металу $p$ ) є порівняно великим. Цей максимум сильно залежить від коефіцієнту деполяризації кору $L_{z}^{(1)}$, коли $L_{z}^{(2)}$ для оболонки залишається постійним і меншим ніж $L_{z}^{(1)}$. Якщо частота зовнішнього випромінювання наближається до частоти поверхневих плазмонів металу, локальне поле в частинці значно зростає. Наголошується, що максимальне значення коефіцієнта підсилення $|A|^{2}$ еліпсоїдального включення стає особливо важливим у випадку, коли діелектричний кор перевищує металеву фракцію включення. Графічно представлені результати числових обчислень для типових малих срібних частинок.

Ключові слова: коефіцієнт підсилення, еліпсоїдальне включення, коефіцієнт деполяризації, локальне поле, резонансна частота 


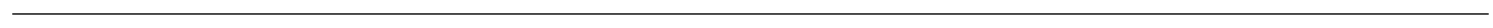

